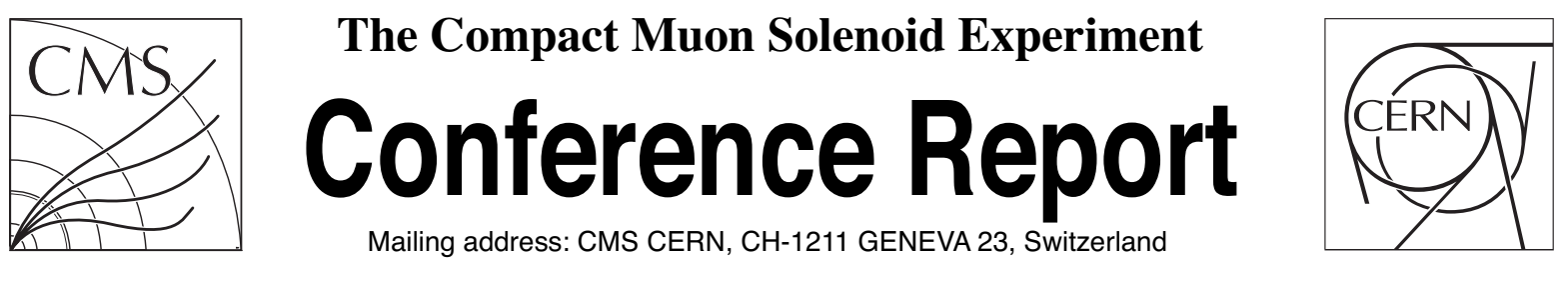

21 November 2008 (v2, 02 December 2008)

\title{
Tracking performance with cosmic rays in CMS
}

\author{
G.B. Cerati on behalf of the CMS collaboration
}

\begin{abstract}
The CMS Tracker is the biggest all-silicon detector in the world and is designed to be extremely efficient and accurate even in a very hostile environment such as that close to the CMS collision point. It consists of an inner pixel detector, made of three barrel layers (48M pixels) and four forward disks (16M pixels), and an outer micro-strip detector, divided in two barrel sub-detectors, TIB and TOB, and two endcap sub-detectors, TID and TEC, for a total of 9.6M strips. The commissioning of the CMS Tracker detector has been initially carried out at the Tracker Integration Facility at CERN (TIF), where cosmic ray data were collected for the strip detector only, and is still ongoing at the CMS site (LHC Point 5). Here the Strip and Pixel detectors have been installed in the experiment and are taking part to the cosmic global-runs. After an overview of the tracking algorithms for cosmic-ray data reconstruction, the resulting tracking performance on cosmic data both at TIF and at P5 are presented. The excellent performance proves that the CMS Tracker is ready for the first collisions foreseen for 2009.
\end{abstract}

Presented at 11th Topical Seminar on Innovative Particle and Radiation Detectors (IPRD08),1-4 October 2008,Siena,Italy,30/11/2008 


\section{INTRODUCTION}

The evaluation of the track reconstruction performance on real data is crucial to check the detector and reconstruction status, measure inefficiencies and understand detector calibration or alignment problems. The first chances to test the track reconstruction performace in CMS were the cosmic runs at the Tracker Integration Facility[2], where part of the strip detector was setup, and at the CMS site, with the full tracker detector installed within the experiment in its final configuration.

\section{THE CMS TRACKER}

A robust tracking combined with a precise vertex reconstruction is crucial to address the physics challenge of proton collisions at LHC. An all-silicon tracking system[1] with very fine granularity has been built and now is in the final commissioning phase. It represents the largest silicon tracking detector ever built. The system is composed by two pixel detectors (barrel and forward) and four micro-strip detectors (Tracker Inner Barrel, TIB; Tracker Outer Barrel, TOB; Tracker Inner Disks, TID; Tracker End-Cap, TEC). The CMS tracker provides full coverage for $|\eta|<2.4$ with more than 10 high-resolution measurement points, among which at least 5 provide a 3-dimensional position measurement.

The tracker detector commissioning was performed in several stages and in different places for the various parts of the detector. The assembly of the four strip sub-detectors was carried out at the CERN Tracker Integration Facility (TIF) in 2006 and 2007, where the first runs on cosmic ray data were performed. The barrel pixel detector was built, assembled and tested at PSI (Zurich), while the forward pixel detector was built and assembled at FNAL (Chicago) and later commissioned at TIF. With the strip detectors already installed since December 2007, during Summer 2008, the pixel detectors were inserted into the CMS experiment at LHC Point 5 and the commissioning operations of the whole tracker detector with cosmic ray data started.

\section{TRACKING ALGORITHMS FOR COSMIC DATA}

The CMS tracking group has developed a complex tracking strategy that leads to optimal track reconstruction performance for any physics object use case [3]. In particular, three algorithms have been develped for cosmic track reconstruction [4]. Two of them, namely the Combinatorial Kalman Filter and Road Search, are "general purpose" algorithms adapted to reconstruct cosmic muons, the other one, called Cosmic Track Finder, is a dedicated algorithm for this kind of events.

\subsection{Combinatorial Kalman Filter}

Combinatorial Kalman Filter (CKF) [5] is the default algorithm for track reconstruction in CMS. Starting from a seed, it searches for the track hits following the track direction using a Kalman Filter approach [6]. In the default configuration, the seed is made of hit pairs or triplets located on the innermost tracker layers. For cosmic tracks, instead, seeds are built from hits on the outermost layers in the top half of the tracker. In addition, once the seeds are found, the pattern recognition is performed applying looser cuts with respect to those used for LHC collision tracks.

\subsection{Road Search}

Road Search (RS) has a different approach with respect to CKF: RS seeds have hits both in the inner and outer layers; "roads" connecting the seed hits are drawn and, starting from low occupancy layers, the most compatible hits along the road are collected. As expected for collision tracking, by default RS reconstructs tracks in one half of the tracker only; in the version for cosmics, instead, if two matching track-segments are reconstructed in opposite tracker halves, they are merged in one single track.

\subsection{Cosmic Track Finder}

Cosmic Track Finder (CosmicTF) is a dedicated algorithm for the reconstruction of cosmic tracks. It is designed to reconstruct only one track per event. All pairs of hits in the outer layers are used as seeds, and each seed corresponds to a trajectory candidate. Trajectory candidates are iteratively propagated to all the hits in the tracker according to their order with respect to the vertical coordinate. If a hit is compatible, then it is added to the 
trajectory. After all seeds have been processed, the resulting tracks are analyzed and only the best track, according to the highest number of hits and the lowest $\chi^{2}$, is kept.

\section{TIF RESULTS}

A slice of the strip tracker, composed of 2161 modules, $\sim 15 \%$ of the four sub-detectors, was sandwitched between scintillator counters providing trigger coincidence signal (Fig. 1). A $5 \mathrm{~cm}$ thick lead plate, placed on top of lower scintillators, served as shield from tracks with momentum below $200 \mathrm{MeV}$. During the runs, the operating temperature was gradually decreased from $+15^{\circ} \mathrm{C}$ to $-15^{\circ} \mathrm{C}$. At TIF, about 5 million events were collected and processed to reconstruct tracks [4]. The total number of collected tracks was about 2.3M. Similar performance results were obtained for the three tracking algorithms used (CKF, RS, CosmicTF). Many analysis methods, based on tracker data only, were developed during the TIF data-taking. Some of them are here presented.
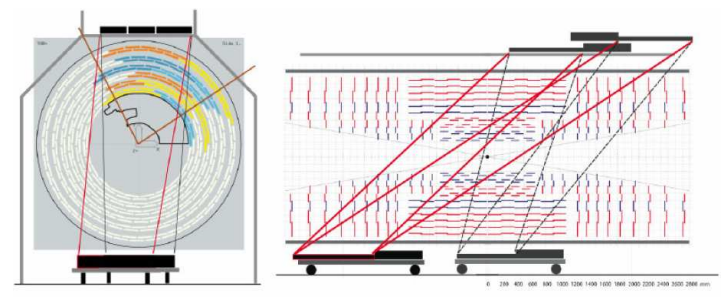

Figure 1: One of TIF trigger layouts. The $x-y$ view is shown on the left side, the r-z view is shown on the right. The straight lines connecting the active areas of the top and bottom scintillation counters indicate the acceptance region. In the $x-y$ view, the active TOB modules are shown in contrasting colors while the active TIB area is framed in black.

\subsection{Tracking efficiency}

In order to measure efficiency, two classes of independently reconstructed tracks were considered: TOB tracks, which are seeded in the outer TOB layers and tracked in the TOB only, and TIB tracks, seeded in the inner TIB layers and tracked in the TIB only. Two efficiencies were computed: $\epsilon(T O B \mid T I B)$, the probability to find a matching TOB track for a given TIB track, and, vice versa, $\epsilon(T I B \mid T O B)$. Tracks were declared matched if their azimuth angles difference was within 5 times the estimated resolution. Only the events with just one reconstructed track are considered. Obtained results are in the range 90\%-99\% (Table 1); the small discrepancies observed with respect to Monte Carlo are due to a different acceptance between reconstructed and simulated data.

Table 1: Tracking efficiency results for the three algorithms, as measured in data and in the Monte Carlo simulation (MC).

\begin{tabular}{c|cc}
\hline$\epsilon(T O B \mid T I B)[\%]$ & Data & MC \\
\hline CKF & $94.0 \pm 0.2$ & $98.3 \pm 0.04$ \\
CosmicTF & $93.1 \pm 0.2$ & $95.6 \pm 0.09$ \\
RS & $89.9 \pm 0.2$ & $92.7 \pm 0.12$ \\
\hline$\epsilon(T I B \mid T O B)[\%]$ & Data & MC \\
\hline CKF & $97.7 \pm 0.1$ & $98.8 \pm 0.04$ \\
CosmicTF & $97.0 \pm 0.1$ & $99.1 \pm 0.06$ \\
RS & $99.0 \pm 0.1$ & $99.4 \pm 0.03$ \\
\hline
\end{tabular}

\subsection{Momentum estimation from multiple scattering}

At TIF, the track momentum could not be directly measured because no magnetic field was present. The cosmic momentum distribution was estimated with a method based on multiple scattering: in fact, most of cosmic tracks have low momenta and thus their direction is dominated by multiple scattering. The direction uncertainty introduced at each crossed layer depends on the particle momentum and the track momentum can be evaluated by refitting the track with the momentum value floating and requiring a fit $\chi^{2}=1$. Measured distribution is in good agreement with Monte Carlo. 


\subsection{Energy loss measurement}

The analog readout of the CMS tracker allows for the measurement of the energy lost by a particle crossing a tracker layer $\Delta E$. The energy loss per unit pathlength is a valuable estimator for particle identification. It can be computed as $d E / d x=\Delta E /(\Delta L \cdot \sec \theta)$, where $\Delta L$ and $\theta$ are the silicon module tickness and the angle between the track direction and the axis perpendicular to the module surface. Given the trajectory of a charged particle, it is possible to measure the energy deposited in the different layers of the tracking system and to attribute a single $d E / d x$ to the track. Results show that measured $d E / d x$ distribution is compatible with expectations for MIPs.

\section{CRUZET RESULTS}

CRUZET, acronym for Cosmic RUn at ZEro Tesla, is the first global run making use of full CMS detector, trigger and Data Quality Monitor (DQM). The tracker detector joined the CRUZET3 data-taking (7-14 July 2008) with the strip detector only, and the CRUZET4 (18-25 August 2008) with both the strip and pixel detectors. The tracker operated in the same conditions foreseen for the collision runs: the raw data from the Front-End Driver (FED) boards are collected and promptly reconstructed; these data are then processed updating the alignment and calibration contants and finally re-reconstructed making use of the new conditions. During these global runs, the tracking algorithms were used to reconstruct tracks in the whole tracker detector for the first time. For standalone tracker analyses, CRUZET performance was evaluated using the methods developed at TIF, while, exploiting data from other detectors, new analysis techniques were also implemented.

\subsection{Tracking efficiency}

The tracking efficiency has been evaluated through two different methods: In the first one, tracks are reconstructed in the muon chambers and, for those pointing to tracker volume, a matching tracker track is looked for. The second method is based on the TIF tracking method and the efficiency is evaluated using tracks reconstructed in the upper and lower halves of the tracker (Fig. 2). The two methods provide consistent results. In particular, the estimated global efficiencies are at the level of $85 \%-90 \%$ with a peak around $95 \%$ for vertical tracks in the central tracker. They are in reasonable agreement with Monte Carlo predictions.

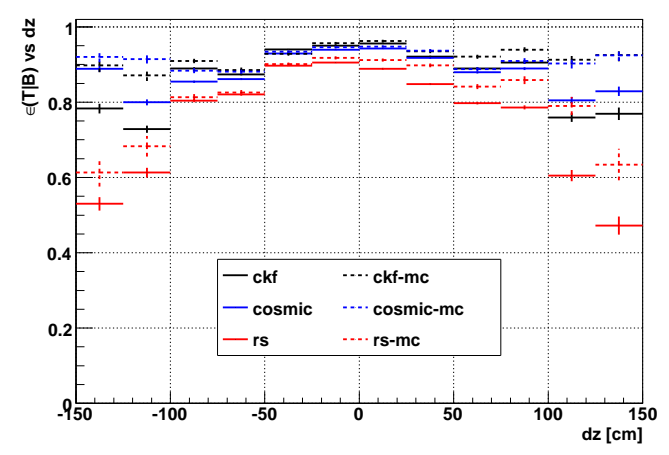

Figure 2: Tracking efficiency using tracker data only $(\epsilon($ Top $\mid$ Bottom $))$ as a function of the longitudinal impact parameter $d z$ for CKF (black), CosmicTF (blue) and RS (red). CRUZET data are solid lines, MC are dashed.

\subsection{Hit efficiency and resolution}

Hit efficiency and resolution are computed by reconstructing tracks excluding the information from a particular module. The reconstructed tracks are then extrapolated to the considered module. Hit efficiency is then computed as the fraction of times a compatible hit is found on the module. In order to avoid artificial inefficiencies, a fiducial area on the module was defined. As expected, for most modules the efficiency is close to $100 \%$; nevertheless, modules with low efficiency have been found, providing a cross-check of the inactive module list and helping to solve a cabling map error.

The hit resolution is estimated by comparing the measured hit position with the one predicted by track extrapolation. The resolution is computed for the overlapping tracker layers, where the amount of material and the propagation distance between layers are minimal. It avoids translational misalignment problems by computing a 
double difference between the hit positions and predictions on the two overlapping layers. This method provides resulting resolutions of $\sim 20 \mu \mathrm{m}$ for TIB and $\sim 40 \mu \mathrm{m}$ for TOB, compatible with the expectations.

\section{CONCLUSIONS}

Great progresses have been accomplished in understanding the performance of the CMS tracker. Cosmic runs at TIF allowed for the first studies of CMS tracking with real data, testing reconstruction algorithms and developing analysis methods. After the tracker was installed within the experiment, it joined CMS cosmic global runs and took data for several weeks in a LHC-ready configuration. The analysis of these data have been used to evaluate tracking performance and check the detector status. Results are remarkable, particularly considering that the detector was still under commissioning, and are in good agreement with Monte Carlo, proving that the understanding of the real detector is already at a very good level. They demonstrate that the CMS tracker detector is ready for LHC collision.

\section{ACKNOWLEDGMENTS}

The author would like to thank the CMS tracking group and the conference organizers.

\section{References}

[1] R. Adolphi et al., The CMS experiment at the CERN-LHC, JINST 3:S08004, 2008.

[2] W. Adam et al., Silicon Strip Tracker Detector Performance with Cosmic Ray Data at the Tracker Integration Facility, CMS NOTE-2008/032.

[3] P. Azzurri, Track reconstruction in $C M S$, talk given at this conference.

[4] W. Adam et al., Track Reconstruction with Cosmic Ray Data at the Tracker Integration Facility, to be published on JINST.

[5] CMS Collaboration, CMS Physics TDR Volume 1, CERN-LHCC-2006-001 (2006).

[6] R. Fruhwirth, Application of Kalman filtering to track and vertex fitting, Nucl.Instrum.Meth.A262:444-450, 1987. 